\title{
Sustainable Imaging Technology for Thermal Printing
}

\author{
Terri Powell ${ }^{1}$, Brian Einsla ${ }^{2}$ and John Roper $^{3}$ \\ 1. The Dow Chemical Company, Analytical Sciences, Collegeville, PA, USA. \\ 2. The Dow Chemical Company, Functional Coatings, Collegeville, PA, USA. \\ 3. The Dow Chemical Company, Formulation Sciences, Midland, MI, USA.
}

Traditional thermal printing, commonly used for point-of-sale receipts, uses a mechanism that involves a developer and a colorless dye, which changes colors when exposed to the heat of the thermal printhead. Bisphenol A (BPA) is often used as the developer. BPA is also commonly used in polycarbonate food and beverage packaging, where small amounts may leach out of packaging and into the contents, and then be consumed. Because BPA weakly mimics the hormone estrogen, it has become a material of concern for consumers. Although recent studies by the FDA indicate that there may be little or no health issues associated with BPA, a new thermal printing technology was developed which addresses those consumer concerns.

The new printing method uses a physical change, rather than chemical reaction, as the mechanism for printing. Hollow spherical particles, also known as "opaque polymers," can serve a variety of functions depending on the void size and/or shell thickness, and can be optimized for properties including crush resistance, scattering/opacity, and insulation. In this new mechanism for thermal printing, a colored layer is applied to the base paper, with a coating of opaque polymer particles on top. When the particles are intact, the light scattering ensures that this layer is opaque. When the paper is exposed to the heat of the thermal printhead, the opaque polymer particles collapse. On collapse, they lose their ability to scatter light and become transparent, revealing the colored layer beneath. A schematic comparing the existing and new print mechanisms is shown in Figure 1.

The challenges for this new print mechanism include maintaining opacity prior to printing, ensuring that particle collapse only occurs under controlled conditions, and providing adequate print contrast. Additionally, this mechanism must be compatible with existing thermal printing hardware. All of these challenges were met through optimization of the particle morphology and coat weight, resulting in good contrast after printing. Characterization of the particles and confirmation of the particle collapse mechanism were conducted by Scanning Electron Microscopy (SEM), as shown in Figure 2.

This innovation in thermal printing offers an alternative mechanism to the traditional chemical print mechanism, and provides phenol-free point-of-sale receipts. It is the first thermal paper approved for food contact. Consumers can easily distinguish it from traditional thermal paper based on color, and it provides better aging with UV stability, resulting in a longer lasting image. It uses the same printing hardware as the current printing technology, so no investment in new hardware is required.

This new printing method was developed in a collaboration between The Dow Chemical Company and the Koehler Paper Group, and was the recipient of the 2017 Presidential Green Chemistry Challenge Award for Designing Greener Chemical, two R\&D 100 Awards, a Business Intelligence Group Sustainability Award, and an Edison Award. 

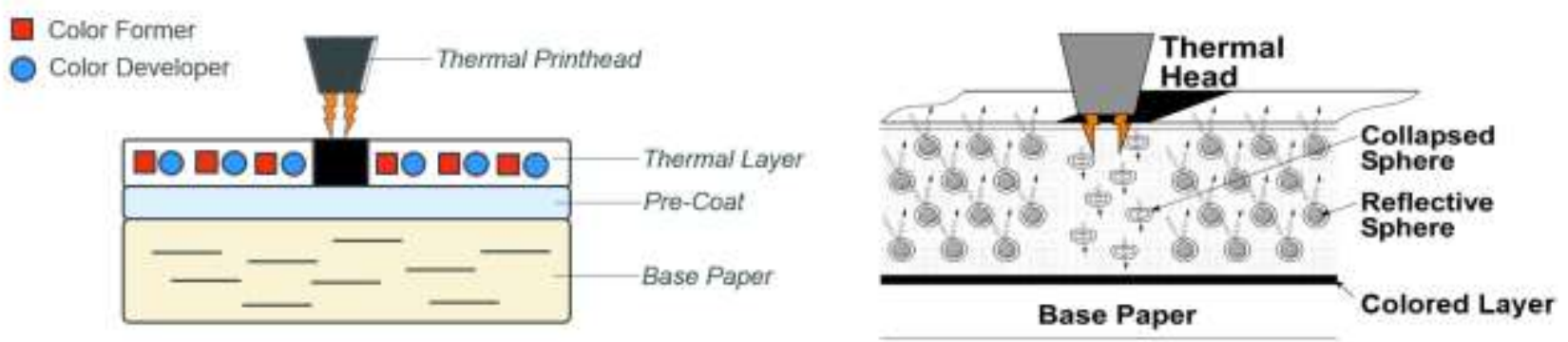

Figure 1: Schematic of mechanism for existing thermal printing (left) and new sustainable thermal printing (right).
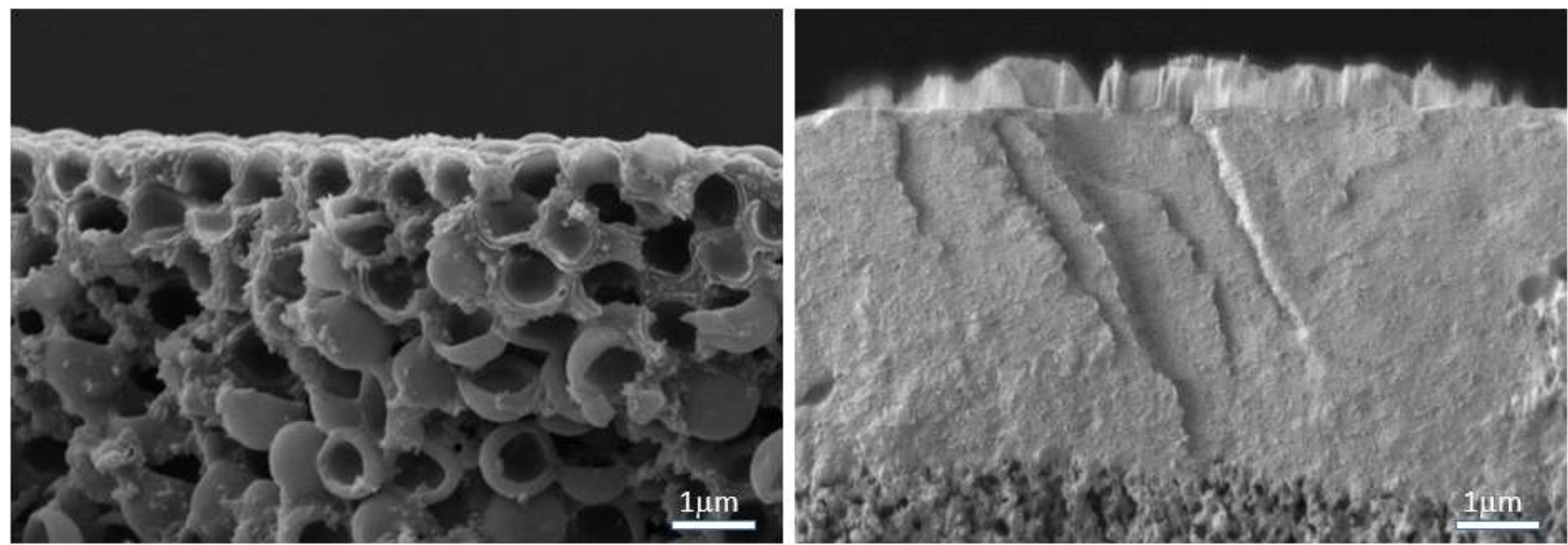

Figure 2: Freeze fractured cross sections of unprinted area (left) and printed area (right) of new thermal printing, showing complete particle collapse in the printed area. 